\title{
The Combination of the Hilbert Transform and the Fourier Transform for Mechanical Vibration Signal Analysis
}

\author{
Xiao Wang ${ }^{1, a}$, Yan Zhu ${ }^{1, b}$ and Bo $\mathrm{Yu}^{2, \mathrm{c}}$ \\ ${ }^{1}$ No. 710 R\&D Institute, CSIC, Yichang 443003 China \\ ${ }^{2}$ Department of Mathematics, College of Science, China Three Gorges University, Yichang 443002 \\ China \\ a email: 15872537001@163.com, bemail:zhuy2006@126.com, \\ cemail:yubo2003@amss.ac.cn. (Corresponding author)
}

Keywords: Empirical Mode Decomposition; Refinement Fourier Spectrum Analysis; Hilbert Transform; Analytic Signal; Instantaneous Phase

\begin{abstract}
In this paper we propose one method for mechanical vibration signal analysis. The given mechanical vibration signal is firstly decomposed into several intrinsic mode functions (IMFs) by the empirical mode decomposition (EMD) method. The Hilbert transform of each IMF, calculated by a recently developed fast algorithm, is then implemented and the analytical signal corresponding to each IMF is obtained. After writing the analytical signal in polar coordinates, we do the refinement Fourier spectrum analysis for the phase function of every analytical signal. By comparing the behavior of these analytical signals, which correspond to different IMFs, in the frequency domain with the characteristic frequency of the mechanical system, we distinguish the IMFs which contain the effective information from the IMFs which consist of noise in the original signal. Numerical results show that the combination of the Hilbert transform and the Fourier analysis works well for practical signals.
\end{abstract}

\section{Introduction}

Since the mechanical systems such as the industry manufacture and the transportation tools play more and more important role in the world, the study of vibration signal generated by mechanical system is very important. The analysis of mechanical vibration signal has a lot of applications in different fields, for example, the fault diagnosis for mechanical systems including machines or some components of machines, the vibration monitoring and control, the production quality control, and the noise source location and noise cancellation.

Generally speaking, the mechanical vibration signal analysis consists of the extraction of information from measured signal patterns. Traditional approach to investigation of mechanical vibration signal can include a spectral analysis based on the Fast Fourier Transform (FFT) [1] and a time-frequency distribution analysis based on the wavelet transform [2]. The classical spectrum analysis method based on FFT has the advantage of high speed but suffers from unsatisfactory understanding of the underlying mechanical system, especially in processing the time-varying vibration signals, such as weak signal submerged by the side lobe of strong signal, low-frequency resolution, and signals corrupted by strong noise. The time-frequency distribution analysis method based on wavelet transform can decompose any signal into a linear combination of a set of basic functions which are dilations and translations of a given mother wavelet. If the mother wavelet has some good properties such as compact support, high vanishing moments and so on, then this decomposition of the signal will present a reasonable time-frequency distribution, from which we can find out the signal's behavior in different frequency bands and at different time durations. However, every time when we try to obtain the time-frequency distribution based on the wavelet transform, we need to choose one suitable mother wavelet, which is, in some sense, empirical.

Currently, the application of Hilbert transform to mechanical vibration signal analysis attracted considerable attention, especially after the advent of the Hilbert-Huang transform, which consists of 
the EMD method and the Hilbert spectrum analysis [3]. For example, some authors combined the EMD method and the wavelet transform to present a fault diagnosis for wind turbine gear box [4]. For more information of the use of Hilbert transform in mechanical vibration signal analysis, please refer to [5]. The success of the Hilbert-Huang transform lies in two facts. The first fact is that, by using the EMD method, it can decompose any signal into a sum of IMFs adaptively and quickly. The second fact is that it can present a global Hilbert spectrum analysis, from which we can easily find out the instantaneous frequency of the analyzed signal. However, there is no need to implement the Hilbert spectrum analysis in mechanical vibration analysis because the main advantage of the processing of mechanical vibration signal is that we actually know some information of the analyzed signal before we analyze it, especially in the frequency domain. For example, when we do fault diagnosis for gear box, we do have some priori knowledge about the behavior in the frequency domain of the signal generated by the defect gear box. Keeping this in mind, we can only use the EMD part of the Hilbert-Huang transform to decompose the signal into several IMFs. These IMFs may contain the noise, the characteristic information of the mechanical system and some other information. The next step is to find out the IMFs which contain the effective information and the IMFs which contain the noise information. For this purpose, we calculate the Hilbert transform of each IMF, from which we obtain the analytic signal corresponding to each IMF. Thus, we can get the instantaneous amplitude function and instantaneous phase function with respect to each IMF. After applying the refinement Fourier spectrum analysis for all these phase functions and comparing them with the characteristic frequency of the underlying mechanical system, we can extract the informative IMFs from the given signal.

This paper is organized as follows. Section 2 describes the algorithms, including the EMD method, the calculation of the Hilbert transform, and the refinement Fourier spectrum analysis. Section 3 presents several numerical results. The conclusion will be drawn in Section 4.

\section{Algorithm Description}

In this section we will describe our algorithm which consists of three steps. The first step is to decompose the given mechanical vibration signal into several IMFs by using the EMD method. The second step is to implement the Hilbert transform on these IMFs so that we get the corresponding analytic signal, from which the phase functions are obtained. Using the refinement Fourier spectrum analysis, the third step is to find out the effective IMFs according to the characteristic frequency of the mechanical system.

The EMD method was proposed by Huang et al. [3] in 1998. It is a sifting process based on the local oscillation of the signal. The main advantage of this method is that it does not need to pick up any basis functions, which is necessary by the conventional method such as Fourier method and wavelet transform method. This advantage makes the EMD method beyond the choice of different basis functions, which depends on different signals to be dealt with. By this method, the signal will be decomposed into a finite sum of IMFs and a residue, where the IMFs should satisfy: (i). The number of the extrema and the number of the zero crossings are equal or differ at most by one; (ii). At any point, the mean value of the envelopes defined by the local extrema is zero. The detailed algorithm of EMD method can be found in [3]. From the view of mathematics, we can say that the IMFs are adaptive basis functions for some function space. Thus, the EMD method actually presents an adaptive function decomposition method algorithmically.

After the sifting process, the original signal will be decomposed into several IMFs. The next step is to find out the IMFs which contain the effective information and the IMFs which contain the noise information. Generally this is not an easy task. However, the main advantage of the mechanical vibration signal analysis is that we have some priori knowledge about the exact characteristic information, in the frequency domain, of the given mechanical vibration signal. Thus, the Fourier spectrum analysis for these IMFs will be applied. However, before this procedure, we will try to "separate" the amplitude part and phase part of these IMFs to get a more meaningful Fourier spectrum analysis. For this purpose, we calculate the Hilbert transform of these IMFs in next step. 
In [6], the authors developed a fast algorithm for computing the Hilbert transform of a function from a data set consisting of $\mathrm{n}$ function values and proved that the complexity of the proposed algorithm is $O(n \log n)$. The basic idea of that algorithm is to use the fact that the Hilbert transform of B-splines keeps the same recurrence relation as that of B-splines themselves, which was observed in [7] and generalized to multivariate case in [10]. Thus we call this method by the B-spline method for computing the Hilbert transform. Both theoretical proof and numerical results show that the B-spline method has a high computational speed and a low computational error. Therefore, we will use the B-spline method to compute the Hilbert transform of the IMFs in this paper. For the convenience of the reader, let us review the central idea of the B-spline method for computing the Hilbert transform.

Let $k \in N$, and $\mathrm{T}:=\left\{\tau_{j}: j \in \mathrm{Z}\right\}$ be a biinfinite sequence of strictly-increasing real numbers such that $\lim _{j \rightarrow \pm \infty} \tau_{j}= \pm \infty$. For $j \in \mathrm{Z}$, the $j$-th B-spline of order $\mathrm{k}$ for the knot sequence $\mathrm{T}$, denoted by $B_{j, k}$, is defined at $t \in R$ as

$B_{j, k}(t):=\left(\tau_{j+k}-\tau_{j}\right)\left[\tau_{j}, \ldots, \tau_{j+k}\right](\cdot-t)_{+}^{k-1}$,

where $\left[\tau_{j}, \ldots, \tau_{j+k}\right] f$ denotes the $k$-th divided difference of a function $f$ at knots $\left\{\tau_{j}: j \in Z_{k+1}\right\}$ and the dot “." indicates the variable in which the divided difference is computed. For every biinfinite vector $c=\left(c_{j}: j \in Z\right) \in R^{Z}$, we define a spline function $S$ of degree $k-1$ with knots on $\mathrm{T}$ at $t \in R$ as

$$
S(t)=\sum_{j \in Z} c_{j} B_{j, k}(t) \text {. }
$$

For a given data set, if we choose reasonable $c_{j}$ 's according to the data set, then (2) can be interpreted as a B-spline approximation $S$ to a function $f$ underlying the given data set. Thus, by the linear property of the Hilbert transform, at almost everywhere $t \in R$ we have that

$$
(H S)(t)=\sum_{j \in Z} c_{j}\left(H B_{j, k}\right)(t)
$$

For simplicity we set $H_{j, k}=H B_{j, k}$. Thus, the calculation of $H S$ reduces to computing the $H_{j, k}$, which can be implemented efficiently because for $t \in R \backslash \mathrm{T}, H_{j, k}$ 's satisfy the following recurrence relation

$H_{j, k+1}(t)=p_{j, k}(t) H_{j, k}(t)+q_{j+1, k}(t) H_{j+1, k}(t)$

where the functions $p_{j, k}$ and $q_{j, k}$ are defined at $t \in R$ by

$$
p_{j, k}(t):=\frac{t-\tau_{j}}{\tau_{j+k}-\tau_{j}}, \quad q_{j, k}(x):=\frac{\tau_{j+k}-t}{\tau_{j+k}-\tau_{j}} .
$$

The initial function $H_{j, 1}$ is given at $t \in R \backslash\left\{\tau_{j}, \tau_{j+1}\right\}$ by

$$
H_{j, 1}(t)=\frac{1}{\pi} \ln \left|\frac{t-\tau_{j}}{t-\tau_{j+1}}\right| .
$$

Using this fact, the Hilbert transform of a given function can be computed efficiently. In practical calculation, we assume that the values of the function $f$ at a sequence of points $\left\{t_{j}: j \in Z\right\}$ are given. We call $\left\{t_{j}: j \in Z\right\}$ the sample points and $\left\{f\left(t_{j}\right)\right\}$ the samples. We first choose the order $k$ of the B-splines and the knots of the B-splines. Then we choose reasonable $c_{j}$ 's so that $S$ is a good approximation to the underlying function $f$ of the given data. For example, in the case that $h=t_{j}-t_{j-1}$ is a constant, if we choose $k=3$ then $\tau_{j}=\frac{1}{2}\left(t_{j-1}+t_{j}\right)$ and

$$
c_{j}=-f\left(\tau_{j}+h / 2\right) / 8+5 f\left(\tau_{j}+3 h / 2\right) / 4-f\left(\tau_{j}+5 h / 2\right) / 8
$$

are good choices. Thus, (3) can be used to approximate the Hilbert transform of a given data set. 
For more details, please refer to [6].

After the calculation of the Hilbert transform of $f$ we can get the corresponding analytic signal $A f=f+i H f$. If we write this in polar coordinates, then we have $A f(t)=\rho(t) e^{i \theta(t)}$, where $\rho(t)=\sqrt{|f(t)|^{2}+|H f(t)|^{2}}$ and $\theta(t)=\arctan \frac{H f(t)}{f(t)}$ are instantaneous amplitude function and instantaneous phase function of $f$ respectively. The next step is to implement the Fourier analysis to the function $e^{i \theta(t)}$, which contains the frequency information of each IMF.

When the analytic signal corresponding to each IMF is obtained, we can implement the Fourier analysis to the function $e^{i \theta(t)}$. Instead of using the general fast Fourier transform (FFT), we choose a similar method with which we can present a more refined Fourier spectrum analysis. To this end, we introduce the discrete time Fourier transform (DTFT). For a continuous time signal $x$, its Fourier transform is defined as:

$\hat{x}(\xi)=\int_{R} x(t) e^{-2 i \pi \xi t} d t$.

Consider a $\delta$ sampling sequence

$x_{s}(t)=x(t)\left[\sum_{n \in Z} \delta(t-n T)\right]$

where $\delta(t-n T)$ is a Dirac function, $T$ is the sample period and $Z$ is the integer set. For an arbitrary continuous function $y$, we have

$\int_{R} y(t) \delta(t-n T) d t=y(n T)$,

thus the Fourier transform of $x_{s}$ is:

$\hat{x_{s}}(\xi)=\sum_{n \in Z} x(n T) e^{-i 2 \pi \xi \xi T}$.

This is the DTFT of the signal $x$. For a discrete time signal $x(n), n \in Z_{n}:=\{0,1, \ldots, N-1\}$, whose

$$
\hat{x}(\xi)=\sum_{n \in Z_{n}} x(n) e^{-i 2 \pi \xi n / \xi_{s}}
$$

Notice that $\hat{x}$ is a continuous function of frequency $\xi$, we can get the continuous spectrum of signal $s$, thus, we can obtain high resolution spectrum in spite of the limitation of sampling point numbers. Thus we call this by the refinement Fourier transforms.

Using equation (12), we can get the refinement Fourier spectrum for the function $e^{i \theta(t)}$, which obtained from the analytic signal corresponding to each IMF. Comparing the refinement Fourier spectrum with the characteristic frequency information of the mechanical vibration signal, it is easy to find out which analytic signal and the associated IMF contains the characteristic frequency information of underlying mechanical system.

\section{Numerical results and discussion}

In this section we will analyze a practical mechanical signal to illustrate the effectiveness of the proposed algorithm. In Figure 1 we present the practical signal, which consists of the effective information in three different frequency bands, the complex background noise and the biological disturbing noise. Figure 2 and Figure 3 present the first to tenth IMFs, decomposed by the EMD method, of the practical signal respectively. From these IMFs, we can also see that the frequency band of the IMFs decreases along with the sifting process. 


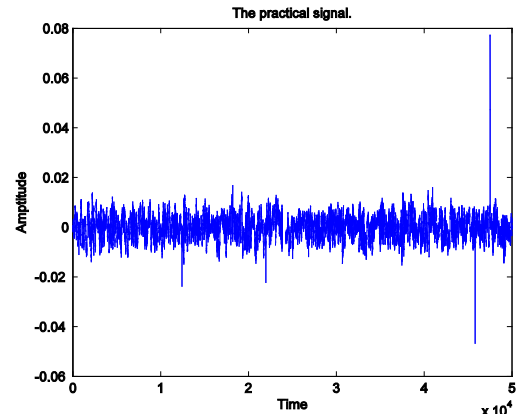

Fig.1. The practical signal

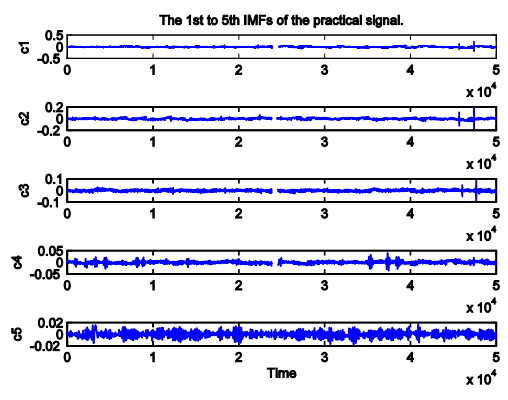

Fig. 2 The first to fifth IMFs

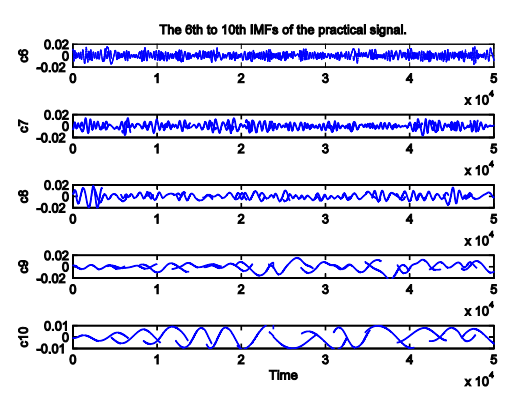

Fig. 3 The sixth to tenth IMFs

The Hilbert transform of the third IMF of the practical signal is showed at Figure 4. Figure 5 gives the refinement Fourier spectrum of the third IMF of the practical signal, from which we can see obviously the frequency amplitude is concentrated around $2438 \mathrm{~Hz}$, which is in the high frequency interval from $1000 \mathrm{~Hz}$ to $10000 \mathrm{~Hz}$.

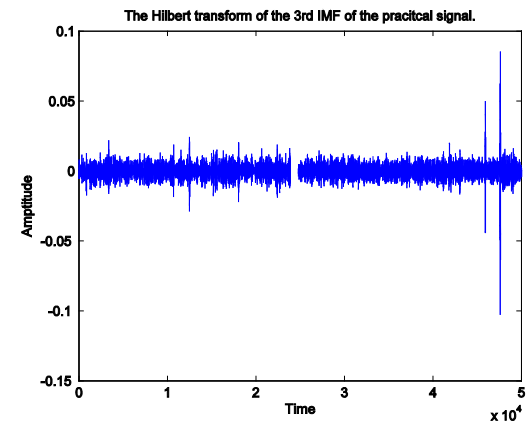

Fig. 4 The Hilbert transform of the third IMF

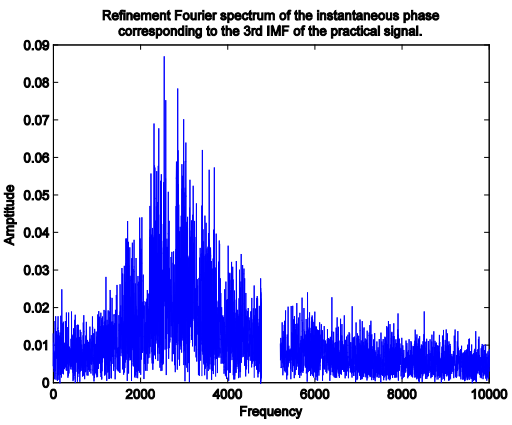

Fig. 5 The refinement Fourier spectrum of the instantaneous phase corresponding to the third IMF

Similarly, the Hilbert transform of the fifth IMF of the practical signal is showed at Figure 6. In Figure 7, we give the refinement Fourier spectrum of the instantaneous phase corresponding to the fifth IMF of the practical signal, from which we can see again the fifth IMF contains the main energy of the practical signal around the frequency $340 \mathrm{~Hz}$, which is in the middle frequency interval from $100 \mathrm{~Hz}$ to $1000 \mathrm{~Hz}$.

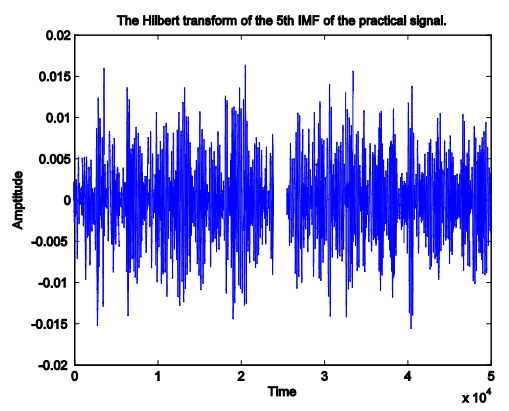

Fig. 6 The Hilbert transform of the fifth IMF

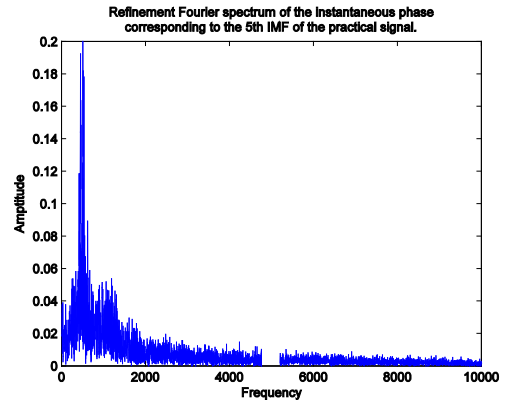

Fig. 7 The refinement Fourier spectrum of the instantaneous phase corresponding to the fifth IMF

Finally, the Hilbert transform of the seventh IMF of the practical signal is shown at Figure 8. In Figure 9, we give the refinement Fourier spectrum of the instantaneous phase corresponding to the seventh IMF of the practical signal, from which we can see the seventh IMF contains the main energy of the practical signal around the frequency $92 \mathrm{~Hz}$, which is in the low frequency interval from $10 \mathrm{~Hz}$ to $100 \mathrm{~Hz}$. 


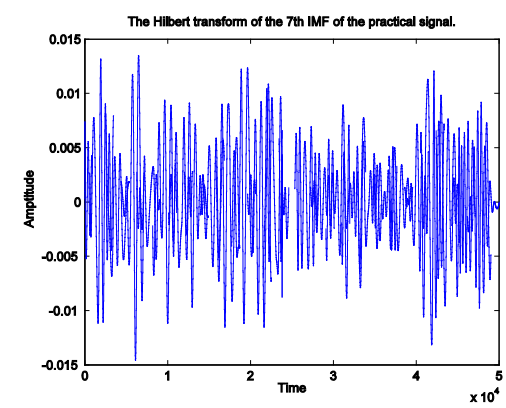

Fig. 8 The Hilbert transform of the seventh IMF

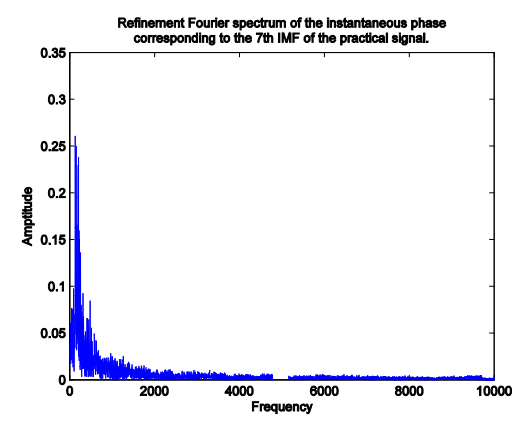

Fig. 9 The refinement Fourier spectrum of the instantaneous phase corresponding to the seventh IMF

From the numerical results shown above, we can see the proposed method works well for practical signals.

\section{Conclusion}

A combination of the EMD method and the refinement Fourier spectrum analysis of the instantaneous phase function presents a reasonable analysis for mechanical vibration signals. The calculation of the Hilbert transform of the extracted IMFs and the refinement Fourier spectrum analysis of corresponding instantaneous phase function help us to understand the underlying mechanical vibration system. This understanding can help us to do further analysis for the mechanical vibration signals. Numerical results confirm the effectiveness of the proposed method in this paper.

\section{Acknowledgement}

This work is supported by the China National Science of Foundation under grant the NSFC 11301296 and the third author is partially supported by the China Scholarship Council. Finally we thank the referees for their time and comments.

\section{References}

[1] L. Cohen, Time-Frequency Analysis, Prentice-Hall, Englewood Cliffs, NJ, 1995.

[2] S. Mallat, A Wavelet Tour of Signal Processing: The Sparse Way, Third Edition, Academic Press, 2008.

[3] N. E. Huang, et al., The empirical mode decomposition and the Hilbert spectrum for nonlinear and non-stationary time series analysis, R. Soc. Lond. Proc. Ser. A Math. Phys. Eng. Sci., 1998 454, 903-995.

[4] Q. Yang and D. An, EMD and wavelet transform based fault diagnosis for wind turbine gear box, Adv. Mech. Eng., 2013, Article ID 212836.

[5] M. Feldman, Hilbert transform in vibration analysis, Mech. Syst. Signal Pr., 201125 (3), 735-802.

[6] C. A. Micchelli, Y. Xu and B. Yu, On computing with the Hilbert spline transform, Adv. Comput. Math., 2013 38, 623-646.

[7] Q. Chen, N. Huang, S. Riemenschneider and Y. Xu, A B-spline approach for empirical mode decomposition, Adv. Comput. Math., 2006 24, 171-195.

[8] C. A. Micchelli, Y. Xu and H. Zhang, On the translation invariant operators which preserve the B-spline recurrence, Adv. Comput. Math., 2008 28, 157-169. 